\title{
Carnets
}

Revue électronique d'études françaises de l'APEF

Deuxième série - 16 | 2019

Le Récit inachevé: études sur Mai 68

\section{Des « Fins de L'homme » à la Naissance des Fantômes ou comment prendre la parole?}

Ana Paiva Morais

\section{(2) OpenEdition}

1 Journals

Édition électronique

URL : http://journals.openedition.org/carnets/9674

DOI : 10.4000/carnets.9674

ISSN : 1646-7698

\section{Éditeur}

APEF

Référence électronique

Ana Paiva Morais, «Des «Fins de L'homme » à la Naissance des Fantômes ou comment prendre la parole? », Carnets [En ligne], Deuxième série - 16 | 2019, mis en ligne le 31 mai 2019, consulté le 08 novembre 2019. URL : http://journals.openedition.org/carnets/9674 ; DOI : 10.4000/carnets.9674

Ce document a été généré automatiquement le 8 novembre 2019.

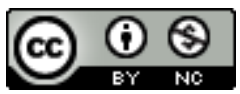

Carnets est mis à disposition selon les termes de la licence Creative Commons - Atribution - Pas d'utilisation commerciale 4.0 International. 


\title{
Des « Fins de L'homme » à la Naissance des Fantômes ou comment prendre la parole?
}

\author{
Ana Paiva Morais
}

\section{« Les Fins de l'Homme»}

Dans le préambule de sa conférence sur «les fins de l'homme», Jacques Derrida affirmait que «tout colloque philosophique a nécessairement une signification politique» (Derrida, 1972:131), et il concluait en soulignant les circonstances politiques qui l'entouraient au moment de l'écriture de ce texte :

[Je voulais] rappeler pour commencer en parlant de la forme de la démocratie comme milieu politique de tout colloque international de philosophie. Et c'est aussi pourquoi je proposais de faire porter l'accent sur forme non moins que sur démocratie. Telle est, dans son principe le plus général et le plus schématique, la question qui s'est imposée à moi pendant la préparation de cette rencontre, depuis l'invitation et la délibération qui a suivi jusqu'à l'acceptation puis la rédaction de ce texte que je date très précisément du mois d'avril 1968 : ces semaines furent aussi, on s'en souvient, celles de l'ouverture des pourparlers de paix au Vietnam et de l'assassinat de Martin Luther King. Un peu plus tard, au moment où je dactylographiais ce texte, les universités de Paris étaient, pour la première fois à la demande d'un recteur, envahies par les forces de l'ordre social, puis réoccupées par les étudiants dans le mouvement d'ébranlement que vous savez. Cet horizon historique et politique appellerait une longue analyse. Les circonstances historiques et politiques dans lesquelles j'ai préparé cette communication, j'ai cru simplement devoir les marquer, les dater et vous en faire part. Elles me paraissent appartenir de plein au champ et à la problématique de notre colloque (Derrida, $1972: 135)^{1}$

2 La date de conclusion de la rédaction est très exactement le 12 mai 1968, et la question majeure que Derrida propose de développer, énoncée, à sa façon habituelle, comme une longue interrogation qui se construit au moment où elle est posée, est une question politique aussi bien que philosophique : « où en est la France quant à l'homme? ». 
3 L'argumentation de Derrida pose d'emblée la question de l'unité de l'homme: malgré l'humanisme affiché de la pensée française après la guerre - il suffit de penser à l'affirmation de Sartre selon laquelle l'existentialisme est un humanisme - il n'en reste pas moins que la notion d'homme n'a jamais effectivement été interrogée quant à son unitarisme fondamental, selon l'auteur, et cela malgré les «suspensions de toutes les présuppositions qui constituaient depuis toujours le concept de l'unité de l'homme [...] que s'imposaient comme un héritage fidèle de la phénoménologie transcendantale de Husserl et l'ontologie fondamentale de Sein und Zeit ", affirme-t-il en s'autorisant de certaines lectures de ces auteurs (Derrida, 1972: 136). Or, comme Derrida ira le démontrer au cours de son essai, ces lectures partielles négligeaient des points importants de la pensée en question que la vague antihumaniste et antianthropologiste qui suivit n'avait pas réussi à vaincre. Tout au contraire, en redécouvrant les écrits de ces auteurs et en s'engageant dans la critique de l'humanisme et de l'anthropologisme, la pensée française "actuelle » n'avait rien fait d'autre que d'«amalgamer Hegel, Husserl et Heidegger avec la vielle métaphysique humaniste » (Derrida, $1972: 141)$.

4 La discussion menée dans ce texte ne met donc pas l'accent sur la question de l'homme, dont les pièges sont reconnus, mais sur ce que Derrida appelle la relève de l'homme, qu'il décrit en se basant sur la pensée de Hegel, de Husserl et de Heidegger. Il prend bien soin de préciser le sens de ce mot traduit de l'Aufhebung de Hegel: "Aufhebung, c'est relever, au sens ou 'relever' veut dire à la fois déplacer, élever, remplacer et promouvoir dans un seul et même mouvement.» (Derrida, 1972: 143). C'est dans les pages qui suivent que Derrida développe la question majeure de la finitude de l'homme. Il n'est pas question ici de suivre les détails de la pensée du philosophe en ce qui concerne l'appartenance de ce concept à la Phénoménologie ; il me suffira de dégager ses conséquences principales telles qu'elles sont posées dans ce texte: «[Le] rapport équivoque de relevance marque sans doute la fin de l'homme, l'homme passé, mais du même coup l'accomplissement de l'homme, l'appropriation de son essence. C'est la fin de l'homme fini. La fin de la finitude de l'homme, l'unité du fini et de l'infini. [...] La relève ou la relevance de l'homme est son télos ou son eskhaton.» (Derrida, 1972: 144). Il faut surtout comprendre dans cette discussion, et c'est le point qui m'intéressera ici, que la question de l'être est liée profondément à la prise de parole dans la pensée, c'est-à-dire que la pensée engage toujours le questionnement. La proximité de soi est, donc, toujours, celle du questionnant :

C'est cette présence à soi, cette proximité absolue de l'étant (questionnant) à luimême, cette familiarité à soi de l'étant prêt à entendre l'être, qui intervient dans la détermination du factum, qui motive le choix de l'étant exemplaire, du texte, du bon texte pour l'herméneutique du sens de l'être. C'est la proximité à soi de l'étant questionnant qui le fait choisir comme étant interrogé par privilège. La proximité à soi du questionnant autorise l'identité du questionnant et de l'interrogé. Nous, qui sommes proches de nous-mêmes, nous nous interrogeons sur le sens de l'être. (Ibid. : 150).

5 On peut comprendre, alors, les titres finaux de ce texte comme une systématisation de « l'ébranlement total sur ce qu'[il a] [...] appelé la 'France' ou la pensée française ». Il ne peut y avoir de motivation philosophique sans l'enjeu d'une motivation politique, affirmait Derrida dans l'ouverture de son texte. Ceci justifie qu'il reprenne, en concluant, l'expression «ébranlement total » qu'il avait employée au début pour désigner les événements de mai en France, bien qu'il le fasse maintenant dans le 
contexte philosophique, bien plus complexe, qu'il vient de décrire. Il semble que son argument fondamental est que le politique ne peut s'énoncer qu'à partir de la philosophie. C'est ainsi que l'ébranlement ne peut se produire que dans la proximité et dans la pensée, et qu'au cœur du «mouvement d'ébranlement» provoqué par ces événements est l'ébranlement du propre :

Ce qui s'ébranle peut-être aujourd'hui, n'est-ce pas cette sécurité du proche, cette co-appartenance et cette co-propriété du nom de l'homme et du nom de l'être, telle qu'elle habite et s'habite elle-même dans la langue de l'occident, dans son oikonomia, telle qu'elle s'y est enfoncée, telle qu'elle s'est inscrite et oubliée selon l'histoire de la métaphysique, telle qu'elle se réveille aussi par la destruction de l'onto-théologie? Mais cet ébranlement - qui ne peut venir que d'un certain dehors - était déjà requis dans la structure même qu'il sollicite. Sa marge était en son corps propre marquée. Dans la pensée et la langue de l'être, la fin de l'homme a depuis toujours été prescrite et cette prescription n'a jamais fait que moduler l'équivoque de la fin, dans le jeu du telos et de la mort. Dans la structure de ce jeu, on peut entendre en tous ses sens l'enchaînement suivant : la fin de la pensée de l'être, la fin de l'homme est la fin de la pensée de l'être. L'homme est depuis toujours sa propre fin, c'est-à-dire la fin de son propre. L'être est depuis toujours sa propre fin, c'est-àdire, la fin de son propre. (Derrida, $1972: 161$ ).

\section{Naissance des Fantômes ou l'agencement du sujet dans le silence}

Mais revenons-en à la question de la prise de parole, lieu par excellence de la déchirure violente du propre, dont parlait Michel de Certeau au lendemain de la fureur des événements de mai 68 : «on a pris la parole comme on a pris la Bastille » en est sa célèbre synthèse. Certes, nul ne peut manquer d'apercevoir le silence épais qui couvre la parole actuellement. Il est évident que prendre la parole ne peut plus être tenu pour une revendication tellement les actes de parole sont menacés par une saturation de discours dans le domaine public aussi bien que dans le privé, mais la parole est surtout brisée par une perte du symbolique, et encore plus, par la prévalence d'un langage qui n'appartient à personne - jamais personne ne peut parler dans 'son' langage. Michel de Certeau le savait déjà en 68 quand il affirmait «cette vérité neuve, fragile, qui est l'expérience [des travailleurs], peut leur être enlevée par la contrainte d'un langage dont ils ne sont pas les auteurs. » (Certeau, 1994 : 56).

7 Un langage, donc, qui risque d'être coupé de l'expérience. Voici un héritage que la mondialisation n'a fait qu'élargir. Le silence s'est désormais installé dans le bruit assourdissant des paroles confuses, dans les discours sans correspondance à l'action, dans une extériorité innommable, et qui ne peut donc se dire que par des paroles fausses, mais dont le paradoxe tient dans sa libre circulation et dans son acceptation tacite même quand leur illusion est manifeste.

8 Marie Darrieussecq a mis ce problème en jeu sous la forme du récit dans Naissance des fantômes. Ce texte étrange et beau fait de la quête de la parole une expérience de la fuite et de l'éloignement. Certes, la littérature s'est depuis toujours intéressée à ce thème de l'éloignement, de l'absence comme lieu de la parole. Pourtant, il est ici posé entre la vieille matrice de l'origine de l'écriture et la béance scandaleuse d'un monde qui s'est écroulé. Comment peut-on arriver à la parole dans un monde pareil ? 
9 Un soir un homme descend de son appartement pour acheter du pain. Il ne revient plus. Sa femme l'attend, puis, ne pouvant pas accepter sa disparition, elle le cherche. L'absence s'installe dans sa vie, dans sa pensée, dans son imagination jusqu'à prendre toute la place dans le récit. L'effacement des traces d'une vie, un rétrécissement du monde et de l'espace, une immobilisation du temps se mettent alors en marche progressivement. Dans un sens inverse, la douleur est gonflée, et elle devient la possibilité même d'ouvrir le monde sur ses mystères, sur ses niveaux inimaginables, sur ses secrets. Les fantômes peuvent surgir.

10 C'est alors seulement que le monde peut être investit par l'écriture, dans les moindres détails de la perception, des sensations et des pensées. Un monde et une réalité qui sortent du silence et de la solitude d'une femme. Ceci justifie peut-être l'entourage presque strictement féminin de la narratrice. La recherche du mari engage un espace intermédiaire de l'absence, une imagination de l'absence qui ne peut être soulignée que par des interlocutrices féminines, des projections de la présence féminine en une sorte d'image vide de la narratrice. La parole du quotidien, la banalité des faits divers, les vies à côté donnent l'image d'une réalité devenue inaccessible, inconnue, méconnaissable. Des corps de femme produisant des paroles égarées.

11 Les coups de téléphone à son l'amie Jacqueline, à la belle-mère et à la mère résonnent sur le fond du silence - «l'air devenait de plus en plus palpable. On sentait sous la tiédeur une butée froide, comme une qualité matérielle du silence »-justement pour le remplir de l'effet d'un réel qui n'est plus accessible que par des interpositions. L'appareil du téléphone est l'instrument de l'éloignement par excellence, mais aussi de l'impersonnalité de celle qui appelle afin de suppléer son déficit de présence pour constater, enfin, que ces voix à l'autre bout ne font que reproduire l'absence qui s'est installée dans sa vie :

Je n'ai pas téléphoné tout de suite à la police. J’ai téléphoné à Jacqueline. Je ne lui ai pas parlé du retard de mon mari, je voulais juste (c'était idiot) savoir si par hasard il n'était pas chez elle. Entendre Jacqueline, seulement Jacqueline, et les cris des enfants, et le clapot d'un bain, ça me faisait du bien, la solidité de Jacqueline dans le commencement de mon angoisse, le volume qu'elle occupait, sa découpe dans l'espace comme une hirondelle énergique. Dans le monde qu'habitait Jacqueline on ne pouvait pas disparaître comme ça, en allant chercher le pain. (Darrieussecq, $1998: 17-18)$

12 La dissipation de la réalité se trouve, alors, dans une communication qui s'épuise presque totalement dans l'écoute de l'autre. Mais cette voix écoutée renvoie de moins en moins à un corps qui puisse assurer quelque rapport à la réalité extérieure. La narratrice qui a appelé son amie dans l'espoir de déjouer l'absence, qui avait même imaginé, pour un instant, que son mari pourrait se trouver auprès d'elle et qu'une explication rationnelle était encore possible, découvre, un peu plus tard en répondant à sa mère au téléphone, que cette présence extérieure est en train de perdre sa matérialité elle aussi :

Le téléphone a sonné [...] On me parlait, ma mère qui m'appelait de son bureau. Il m'a semblé qu'il suffirait, pour renouer avec la normalité des choses, de reprendre avec elle notre vielle partie; et tout se remettrait en place, le kaléidoscope se stabiliserait sur la bonne image : moi, mon mari, ma belle-mère, ma mère. [...] J'ai dit: mon mari a disparu. C'était comme tenter une expérience chimique, faire entrer dans un corps un élément étranger. Ma mère a gardé le silence [...] Ton mari, a dit ma mère, et je n'ai plus entendu que le bruissement d'une petite forêt entre nous. Il me semblait que sa voix s'amenuisait, rétrécissait comme un corps matériel 
de plus en plus petit et incongru, un comédon, du cérumen cristallisé tout au bout de la ligne comme au fond d'une oreille. J'ai raccroché. Le jeu était entièrement transformé. (Darrieussecq, 1998 : 57)

13 Et même la visite de Jacqueline n'a pas réussi à replacer la réalité dans l'horizon de la narratrice, comme si la présence de son amie alourdissait encore plus cette immatérialité envahissante : «[elle] eut quelque chose d'irréel» (Darrieussecq, 1998: 73). En même temps qu'elle vient vers la narratrice, son corps devient plus flou jusqu'à se dissoudre dans la brume de l'imagination de celle-ci :

Jacqueline, debout devant moi, continuait de parler, et il me semblait la voir se réduire, tête et membres, comme sous l'action d'un taxidermiste jivaro, se profiler toute petite dans le fond de la pièce alors qu'elle était à un mètre de moi et que je sentais sur mon visage le vent virulent de ses gestes d'oratrice; et sa voix se dédoublait, sonore et pleine d'échos, une autre voix en elle répondait à la première en un harmonieux fil continu. [...] Jacqueline s'arrêta d'elle-même, mal à l'aise, et ce qui continuait à trembloter autour d'elle en fut un instant interdit, comme si les vibrations de sa voix avaient aménagé tout de même, dans l'air, un espace où le vide devenait habitable. (Darrieussecq, 1998 : 75 (...) 78).

C'est après le dernier coup de téléphone, de la belle mère, que le silence s'installe vraiment dans la narratrice. Les femmes qui l'entourent ont suppléé pendant un moment sa voix défaillante par une sorte de cavité résonnante, mais une fois recueillie dans l'espace vide de la maison, elle se retrouve devant la réalité de sa disparition matérielle : «j'étais une tête chercheuse à laquelle tout fait défaut, radar, antennes, écailles, capteurs d'ultrasons et yeux à infrarouge; je sentais se disjoindre épaule, coude, poignet et phalanges, désossés. Je me suis immobilisée." (Darrieussecq, 1998 : 93-94). Et c'est dans ce silence que naîtra l'existence réelle, c'est-à-dire, l'existence dans l'attente du mari, l'existence comme suspension du mari. C'est au cours d'une deuxième visite dans le bureau de celui-ci qui se déclenche chez la narratrice une expérience de l'attente pourvue d'une épaisseur existentielle qu'aucune autre réalité n'aurait pu lui apporter. Cette attente sera dorénavant le lieu de la parole, le sujet de l'écriture. Le sujet de l'œuvre est trouvé, c'est « le récit de mon attente »(Darrieussecq, $1998: 114$ ). « Dans ce retard je reconnaissais le temps que je vivais mieux que si j'avais su l'écrire sans écart » (Darrieussecq : 1998 : 115), se dit-elle.

Ce qui vibrait dans la distance entre l'écriture et ma vie, cette nappe en feuilleté, que j'imaginais aussi mouvante qu'un rayon de soleil lamifié à mes fenêtres, était précisément ce qui me constituait. (Darrieussecq, $1998: 116)$.

On ne s'étonnera pas de voir se développer désormais cette attente comme un imaginaire liquide qui renverse la réalité quotidienne : «l'envers de la rue était comme une remontée de la mer, une nuit d'inondation" (ibid.: 136). Or cette épiphanie de l'imaginaire de l'eau - d'ailleurs très exubérant dans la dernière partie de l'ouvrage en tant que manifestation d'une existence liée à l'attente, a lieu au cours de la fête organisée par sa mère pour son départ et il se décrit en contraste avec cette situation mondaine. Comme si la venue à l'écriture dans l'attente ne pourrait se faire que par un repli sur un ventre maternel libéré de la présence de la mère, un maternel dont l'essence est le lieu.

16 C'est cette parole au fond des océans qui peut dire les fantômes; elle est la seule garantie de la présence à soi dans l'attente. Laisser l'eau monter dans l'homme afin de déloger la mère, l'origine et la fin, pour en finir avec les équivoques de la fin. La prise de parole aujourd'hui, ressemblerait-elle, pour chacun, au récit de son attente? 


\section{BIBLIOGRAPHIE}

CAINE, Philippa (2009). «Redefining the boundaries of corpo-reality for la femme in Marie Darrieussecq's Naissance des fantômes » in Margaret-Anne Hutton (ed.), Redefining the Real. The Fantastic in Contemporary French and Francophone Women's Writing. Oxford, Bern, Berlin, Bruxelles, Frankfurt am Main, New York \& Wien: Peter Lang, p. 45-63.

CERTEAU, Michel de ([1968] 1994). La Prise de parole et autres écrits politiques. Paris : Seuil, « Points essais ».

DARRIEUSSECQ, Marie (1998). Naissance des fantômes. Paris : P.O.L.

Derrida, Jacques (1972). « Les Fins de l'homme », Marges de la Philosophie. Paris : Les Éditions de Minuit, pp. 129-164.

SIRINELLI, Jean-François (2008). Mai 68, l'événement Janus. Paris : Fayard.

\section{NOTES}

1. Tous les italiques dans le corps des citations se trouvent dans l'original.

\section{RÉSUMÉS}

Nous interrogeons ici l'expression " prise de la parole " à partir de quelques textes écrits à des moments proches des événements de mai 68, en particulier l'essai de Jacques Derrida, «Les Fins de L'Homme » (1972) et celui de Michel de Certeau, «La prise de parole » (1968), ainsi que sur les conditions de la prise de parole trente ans après à partir d'une brève analyse de l'œuvre de Marie Darrieussecq, Naissance des fantômes (1998).

In this article I argue that the texts of Jacques Derrida, « Les Fins de l'Homme » (1972) and Michel de Certeau, «La prise de parole» (1968) are a key to the understanding of the expression " prise de parole » such as it was used shortly after the events of May 68 in France, and I question the possibility of the use of this expression in more recent times by a short reading of Marie Darrieussecq's book Naissance des fantômes (1998).

\section{INDEX}

Keywords : prise de parole, ends of man, humanism, absence, ghost

Mots-clés : prise de parole, fins de l'homme, humanisme, absence, fantôme 
AUTEUR

ANA PAIVA MORAIS

IELT NOVA FCSH

anapm[at]fcsh.unl.pt 